\title{
Sinusoidal Obstruction Syndrome, CTCAE
}

National Cancer Institute

\section{Source}

National Cancer Institute. Sinusoidal Obstruction Syndrome, CT CAE. NCI Thesaurus.

Code C143205.

A disorder characterized by severe hepatic injury as a result of the blood vessels of the liver becoming inflamed and/or blocked. 